\title{
Efecto de los ciclos térmicos sobre la ZAT de una soldadura multipasos de un acero inoxidable superdúplex SAF 2507
}

\author{
(Effect of thermal cycles on the HAZ of a stainless steel multipass weld of superduplex SAF 2507)
}

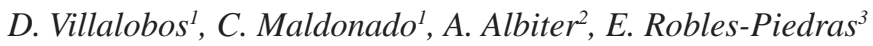 \\ ${ }^{1}$ Instituto de Investigaciones Metalúrgicas, Depto. de Soldadura, Morelia, Michoacán, México, villalobosvd@gmail.com \\ ${ }^{2}$ Instituto Mexicano del Petróleo, Depto. de Aguas Profundas, México, D.F., México. \\ ${ }^{3}$ Instituto de Investigaciones Nucleares, Depto. De Tecnología de Materiales, Ocoyoacac, México.
}

\begin{abstract}
Resumen
Los ciclos térmicos de una soldadura multipasos que experimenta un acero inoxidable superdúplex SAF 2507, pueden promover la precipitación de fases secundarias reduciendo significativamente las propiedades mecánicas y la resistencia a la corrosión. Debido a su aplicación en la industria petroquímica, el estudio de las aleaciones superdúplex es de suma importancia para predecir su comportamiento en servicio cuando están involucrados procesos de soldadura por arco eléctrico. En este trabajo, se estudia el cambio microestructural de la zona afectada térmicamente correspondiente al primer cordón depositado de una unión multipasos de acero inoxidable superdúplex SAF 2507 mediante el proceso GTAW y bajo tres temperaturas de interpasos. Los resultados muestran que la temperatura de interpasos tiene una influencia sobre la precipitación de fase sigma en la zona afectada térmicamente del primer cordón depositado.
\end{abstract}

Palabras-clave: fase sigma, GTAW, ciclo térmico, superdúplex

Abstract: Thermal cycles experienced by a superduplex stainless steel SAF 2507 when is welded, can promote the precipitation of secondary phases which decrease the mechanical properties as well as the corrosion resistance. Due to the application of the duplex alloys in the petrochemical industry, the study of these alloys has become very important in order to predict its service behavior. The aim of this work is to study the microstructural changes in the superduplex stainless steel weld joint after applying the GTAW process under three interpass temperatures after the deposition of every single pass. The results showed that slow cooling rates promoted by the deposition of the subsecuent passes and the higher interpass temperature, promote the precipitation of sigma phase in the HAZ while rapid cooling rates promoted by the lower interpass temperature do not promote the sigma phase precipitation.

Keywords: sigma phase, GTAW, thermal cycle, superduplex

\section{Introducción}

Los aceros inoxidables superdúplex son una excelente alternativa para sustituir a los grados ferríticos y austeníticos debido a que tienen una mayor resistencia mecánica y a la corrosión en medios que contienen cloruros [1]. Debido a que las aleaciones superdúplex tienen una microestructura bifásica de ferrita + austenita en cantidades aproximadamente iguales, sus propiedades de resistencia mecánica y de corrosión están en función de ambas fases, la austenita provee de tenacidad mientras que la ferrita, la cual es una fase de elevada dureza, provee la resistencia mecánica. La cantidad exacta de cada fase está en función de la composición química y del tipo de tratamiento térmico aplicado para su fabricación [2]. Este tipo de aleaciones son utilizadas en la industria del gas y del petróleo así como de la química y petroquímica [3], las cuales han sido desarrolladas para cumplir requerimientos actuales en ambientes de cloruros en cuanto a la resistencia a la corrosión por picaduras o por esfuerzos, ya que contienen cantidades mayores de $\mathrm{Cr}$, $\mathrm{Mo}$

(Recebido em 22/06/2009; Texto final em 20/07/2010). y N [4] en comparación a los grados anteriores. Las aleaciones superdúplex son un acero inoxidable dúplex de alta aleación del tipo $25 \% \mathrm{Cr}-7 \% \mathrm{Ni}-3.2 \% \mathrm{Mo}-0.27 \% \mathrm{~N}$ que tiene un PRE (Pitting Resistance Equivalent) mayor a 40 [4]. Sin embargo, debido a los elevados contenidos de $\mathrm{Cr}$ y Mo y de su elevado rango de difusión en la ferrita, estas aleaciones son susceptibles a formar fases secundarias $(\sigma, \pi, \chi, \mathrm{R}$, carburos, nitruros,etc.) durante la exposición a elevadas temperaturas entre 400 y $1000^{\circ} \mathrm{C}$ [5], como se puede observar en el diagrama Tiempo-TemperaturaTransformación (TTT)de la Figura 1[6]. La fase sigma tiene una importancia particular entre las fases secundarias debido a que pequeñas cantidades causan una considerable reducción en la plasticidad y resistencia a la corrosión en las aleaciones superdúplex [7] debido a que está compuesta preferencialmente por elementos alfágenos como el $\mathrm{Cr}$ y Mo, aunque también se puede observar la presencia de Ni, Si y W si están presentes en la aleación [8]. Palmer et.al., [8) reportaron la precipitación de fase sigma a $850^{\circ} \mathrm{C}$ a partir de 40 segundos en un acero inoxidable dúplex 2205 mientras que Mateo et.al.[9], observaron la precipitación de fase sigma a $650^{\circ} \mathrm{C}$ partir de 3 horas. Para evitar la formación de fase sigma y mantener las propiedades mecánicas y de resistencia a la corrosión en partes o componentes soldados, el balance de fases ferrita-austenita, debe mantenerse tanto en la 
zona afectada térmicamente (ZAT) como en la zona del metal soldadura (MS). La microestructura y propiedades del MS son generalmente controlados por la composición química del material de aporte mientras que en la ZAT se determinan por los ciclos térmicos del proceso de soldadura [10]. En una soldadura multipasos, ciertas regiones de la ZAT y del metal de soldadura, están inevitablemente expuestos al rango de temperatura de formación de fase sigma durante la deposición de los cordones subsecuentes de soldadura, especialmente en la región de la ZAT correspondiente al primer cordón depositado, existiendo una elevada susceptibilidad a la precipitación de fase sigma.

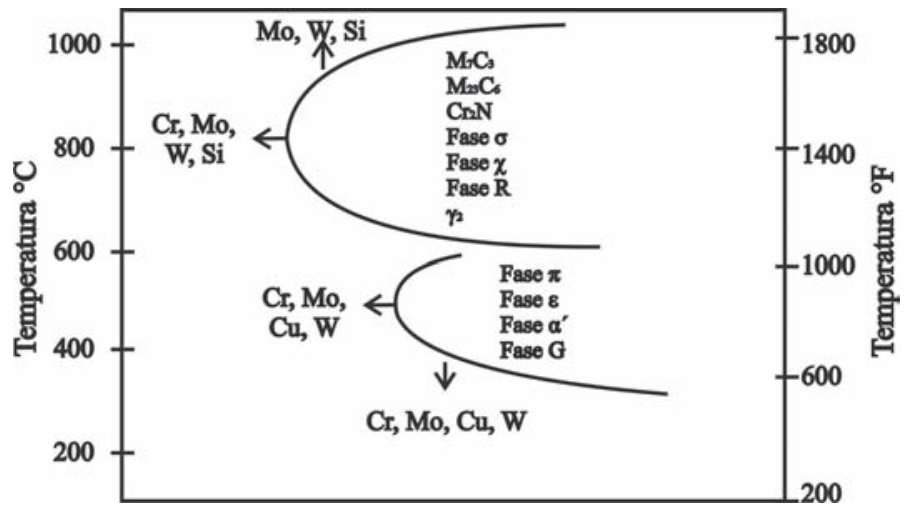

Figura 1. Diagrama de precipitación de fases secundarias en aleaciones dúplex [11]

Durante el proceso de soldadura, la temperatura pico en el MS es superior a la temperatura de fusión del acero inoxidable superdúplex $\left(1483^{\circ} \mathrm{C}\right)$, mientras que en la ZAT correspondiente al primer cordón depositado, las temperaturas pico alcanzadas en esa misma zona en función del número de cordones depositados, están dentro del rango de la temperatura de formación de fase sigma $\left(600^{\circ} \mathrm{C}-1000^{\circ} \mathrm{C}\right)$ por lo que el objetivo principal de este trabajo, es examinar el efecto de los ciclos térmicos experimentados en una soldadura multipasos sobre la ZAT correspondiente al primer cordón depositado mediante el proceso de soldadura por arco con electrodo de tungsteno (Gas Tungsten Arc Welding, GTAW) manteniendo tres temperaturas entre pasadas.

\section{Materiales y Métodos}

Procedimiento de soldadura. Para realizar las uniones se utilizaron placas de acero inoxidable superdúplex SAF 2507 de 6 $\mathrm{mm}$ de espesor para realizar tres uniones soldadas manteniendo una temperatura de interpasos específica en cada una, las cuales fueron preparadas como se muestra en la Figura 2 de acuerdo a lo recomendado por el fabricante de la aleación [12]. El material de aporte fue del tipo superdúplex especificación SANDVIK 25.10.4L de 2.4 mm de diámtetro. El gas de protección fue argón con un flujo de 7 lt/min.

Para realizar las uniones soldadas, fue necesaria la deposición de seis cordones de soldadura los cuales fueron depositados bajo tres condiciones con el objetivo de estudiar la influencia de la velocidad de enfriamiento sobre la unión multipasadas y por lo tanto, modificar los ciclos térmicos a los que se somete el material al aplicar el proceso de soldadura GTAW. Para realizar las uniones multipasadas, la temperatura de cada cordón depositado fue medida mediante un pirómetro y dependiendo de la condición establecida $\mathrm{C} 1\left(25-30^{\circ} \mathrm{C}\right), \mathrm{C} 2\left(100-150^{\circ} \mathrm{C}\right)$ y $\mathrm{C} 3\left(400-450^{\circ} \mathrm{C}\right)$, se aplicaba el siguiente cordón de soldadura. Las condiciones establecidas se muestran a continuación en la Tabla 1 y las variables operativas del procedimiento de soldadura se especifican en la Tabla 2.

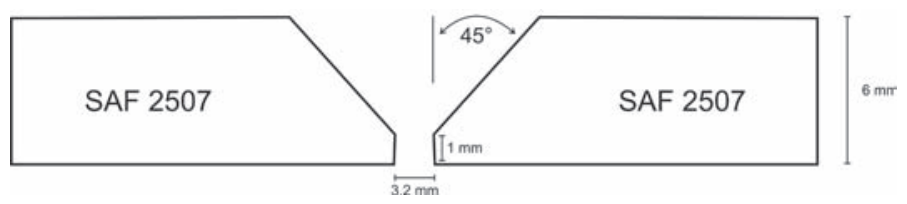

Figura 2. Preparación de la placa soldada.

Tabla 1. Condiciones establecidas para realizar las uniones de soldadura multipasos

\begin{tabular}{|c|c|c|c|}
\hline Muestra & $\begin{array}{c}\text { Cordones } \\
\text { depositados }\end{array}$ & $\begin{array}{c}\text { Temperatura de } \\
\text { interpaso } \\
{ }^{\circ} \mathrm{C}\end{array}$ & $\begin{array}{c}\text { Uniones } \\
\text { experimentales } \\
\text { para cada } \\
\text { condición }\end{array}$ \\
\hline C1 & 6 & $25-30$ & 2 \\
\hline C2 & 6 & $100-150$ & 2 \\
\hline C3 & 6 & $400-450$ & 2 \\
\hline
\end{tabular}

Ciclo térmico. La fase sigma, es la fase que generalmente precipita en la aleaciones dúplex debido a que las temperaturas de formación abarcan un rango de $600-1000^{\circ} \mathrm{C}$ aproximadamente. Por esta razón, es de utilidad conocer la temperatura máxima alcanzada por el material siendo soldado así como el tiempo que permanecerá a dicha temperatura, lo que permitiría estimar las fases presentes en la ZAT de la unión soldada. Para realizar los cálculos necesarios para conocer la temperatura pico y la veloidada de enfriamiento en el rango de $1200-800^{\circ} \mathrm{C}$, se utilizan ecuaciones de flujo de calor. Rosenthal [13], fue el primero en considerar que durante un proceso de soldadura, una fuente de calor en movimiento se aplica sobre la pieza de trabajo para lograr su fusión sin sufrir cambios en la distribución de temperatura y geometría del cordón de soldadura con el tiempo. Para obtener ecuaciones analíticas de flujo de calor, Rosenthal consideró simples suposiciones durante un proceso de soldadura como:

1) Flujo de calor estable

2) Fuente de calor

3) Calor de fusión despreciable

Tabla 2. Variables operativas del proceso de soldadura

\begin{tabular}{|c|c|c|c|c|c|}
\hline $\begin{array}{c}\text { Número de } \\
\text { pasos }\end{array}$ & $\begin{array}{c}\text { Diámetro de Metal de } \\
\text { Aporte (mm) }\end{array}$ & $\begin{array}{c}\text { Intensidad de } \\
\text { corriente (A) }\end{array}$ & $\begin{array}{c}\text { Voltaje } \\
\text { (V) }\end{array}$ & $\begin{array}{c}\text { Flujo de gas } \\
\text { (lt/min) }\end{array}$ & $\begin{array}{c}\text { Velocidad de } \\
\text { Avance (cm/min) }\end{array}$ \\
\hline $1-6$ & 2.4 & 110 & 10 & 7 & $7-8$ \\
\hline
\end{tabular}


4) Propiedades térmicas constantes

5) Sin pérdida de calor de la superficie de la pieza de trabajo

6) Sin convección en la pileta de soldadura

Sin embargo, investigadores como Easterling y Adams [13] han derivado diferentes soluciones a las ecuaciones de Rosenthal y propuesto ecuaciones más simplificadas para la obtención de los cálculos. En este trabajo en particular, se utilizaron las ecuaciones de distribución de calor propuestas por Easterling para calcular la temperatura y la velocidad de enfriamiento de cada una de las soldaduras experimentales. Para calcular el tiempo de permanencia en el rango de $1200-800^{\circ} \mathrm{C}$, se utilizó la ecuación propuesta por Sieurin y Sandström [14]. Por lo tanto, mediante la aplicación de las ecuaciones, se puede calcular la temperatura pico con la ecuación (1) que experimenta el material a una distancia $x$ de la fuente de calor así como la velocidad de enfriamiento en el rango de de $1200-800^{\circ} \mathrm{C}$ desde la temperatura pico mediante la ecuación (2).

$$
\begin{aligned}
& T p-T o=\left(\frac{2}{\pi e}\right)^{1 / 2}\left(\frac{q / v}{d \rho \varsigma 2 r}\right) \\
& \Delta t_{12 / 8}=\Delta t_{8 / 5} \frac{\frac{1}{1073-T o}-\frac{1}{1473-T o}}{\frac{1}{773-T o}-\frac{1}{1073-T o}}
\end{aligned}
$$

donde $q$ es el calor aportado, $v$, la velocidad de avance, $d$, el espesor, $\rho$, el calor específico, $\varsigma$, la densidad, $\lambda$, la conductividad térmica, To, la temperatura inicial o de interpasos. Utilizando las variables operativas del proceso de soldadura mostradas en la Tabla 2 para obtener el calor aportado y las propiedades físicas mostradas en la Tabla 3, se realizaron los cálculos para obtener los ciclos térmicos experimentados por la ZAT en la unión soldada multipasos.

Tabla 3. Propiedades físicas del acero inoxidable superdúplex SAF 2507 (15)

\begin{tabular}{|c|c|c|}
\hline $\begin{array}{c}\text { Conductividad } \\
\text { térmica }(\lambda)\end{array}$ & $\begin{array}{c}\text { Densidad } \\
(\varsigma)\end{array}$ & $\begin{array}{c}\text { Calor específico } \\
(\rho)\end{array}$ \\
\hline $14 \mathrm{~J} / \mathrm{m}^{\circ} \mathrm{C}$ & $7800 \mathrm{~kg} / \mathrm{m}^{3}$ & $480 \mathrm{~J} / \mathrm{kg}^{\circ} \mathrm{C}$ \\
\hline
\end{tabular}

Aplicando la ecuación (1) la temperatura pico para la soldadura experimental C1 (temperatura entre pasos de 25$30^{\circ} \mathrm{C}$ ) a una distancia de $1 \mathrm{~mm}$ desde la fuente de calor y así sucesivamente hasta la distancia de $50 \mathrm{~mm}$, está dada por:

$$
\begin{aligned}
& \mathrm{Tp}-25^{\circ} \mathrm{C}=0.4842 * 2137.78^{\circ} \mathrm{C} \\
& \mathrm{Tp}=1060^{\circ} \mathrm{C}
\end{aligned}
$$

La Figura 3 muestra la gráfica de las temperaturas pico y tiempo de enfriamiento en el rango $1200-800^{\circ} \mathrm{C}$ para cada una de las uniones soldadas bajo las tres condiciones en el rango de distancias de $1 \mathrm{~mm}-50 \mathrm{~mm}$ desde la fuente de calor.

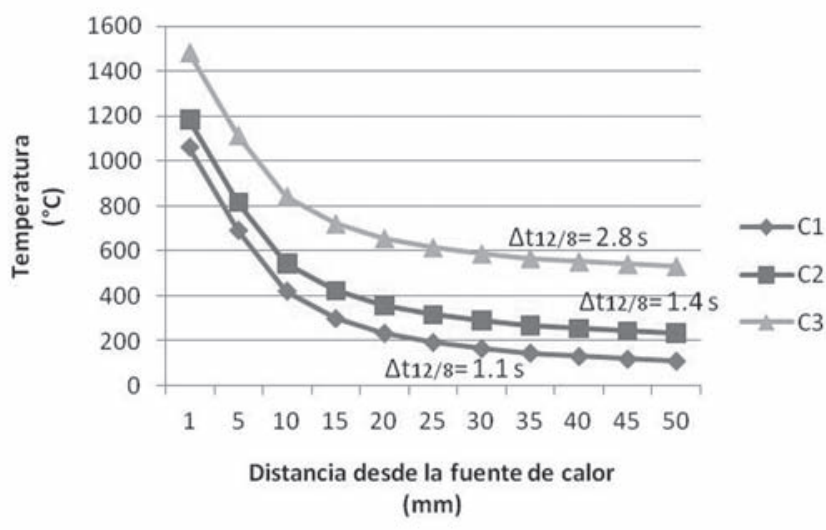

Figura 3. Gráfica de ciclos térmicos calculados para el AISD SAF 2507

Metalografía. La microestructura de la ZAT correspondiente al primer cordón depositado a una distancia de $5 \mathrm{~mm}$ de la fuente de calor, se observó mediante microscopía óptica y microscopía electrónica de barrido. La microestructura fue revelada utilizando un ataque electrolítico. El porcentaje de fases se determinó mediante el programa comercial para análisis de imágenes SigmaScan ${ }^{\mathrm{TM}}$ y se realizaron microanálisis puntuales para obtener la composición química y distribución de elementos en cada fase observada. Para la cuantificación de fases, se realizaron cinco mediciones en cinco imágenes tomadas al azar en las zonas de la unión soldada.

Ensayo de microdureza. Se realizaron mediciones de microdureza Vickers con 50 gr. de carga en cinco granos al azar de la fase ferrita así como de la fase austenita en la zona del metal de soldadura y ZAT.

\section{Resultados y Discusión}

La microestructura inicial del acero inoxidable superdúplex SAF 2507 consta de las fases ferrita y austenita, con un 54\% de ferrita (color obscuro) y un $46 \%$ de austenita (color claro), como se muestra en la Figura 4.

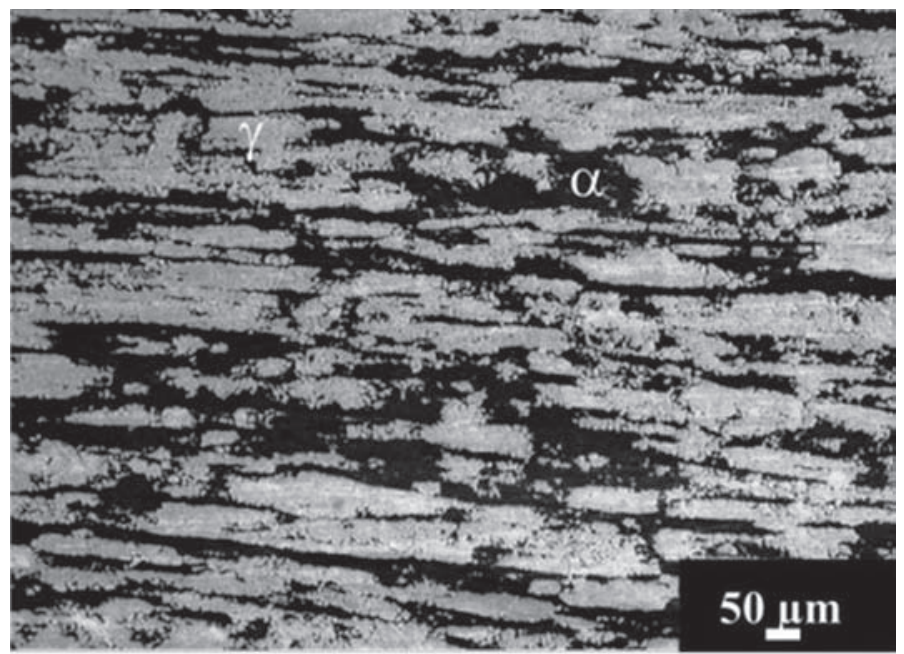

Figura 4. Microestructura inicial del acero inoxidable superdúplex SAF 2507 
Tabla 4. Composición química del material base y del material de aporte (\% en peso).

\begin{tabular}{|c|c|c|c|c|c|c|c|}
\hline MATERIAL & $\mathrm{C}$ & $\mathrm{Si}$ & $\mathrm{Mn}$ & $\mathrm{Ni}$ & $\mathrm{Cr}$ & $\mathrm{Mo}$ & $\mathrm{N}$ \\
\hline SAF 2507 & 0.055 & 0.407 & 0.882 & 5.78 & 23.40 & 3.238 & 0.27 \\
\hline SANDVIK 25.10.4L & 0.060 & 0.490 & 0.509 & 7.32 & 21.72 & 3.009 & 0.25 \\
\hline
\end{tabular}

Tabla 5. Porcentaje de fases en las uniones experimentales.

\begin{tabular}{|c|c|c|c|c|c|c|c|}
\hline \multirow{2}{*}{ Fase \% } & \multicolumn{2}{|c|}{ C1 } & \multicolumn{2}{|c|}{$\mathrm{C} 2$} & \multicolumn{2}{|c|}{ C3 } & \multirow[b]{2}{*}{ MB } \\
\hline & ZAT & CS & ZAT & CS & ZAT & CS & \\
\hline Ferrita & $51_{ \pm 0.83}$ & $57_{ \pm 1.09}$ & $57 \pm 0.83$ & $67 \pm 1$ & $45_{ \pm 0.83}$ & $77 \pm 1.30$ & $54_{ \pm 0.83}$ \\
\hline Austenita & $49_{ \pm 0.1 .30}$ & $43_{ \pm 0.83}$ & $43_{ \pm 1.14}$ & $33 \pm 1.14$ & $52.7 \pm 0.25$ & $23 \pm 0.83$ & $46_{ \pm 0.83}$ \\
\hline Sigma & -- & -- & -- & -- & $2.2 \pm 0.13$ & 0 & -- \\
\hline
\end{tabular}
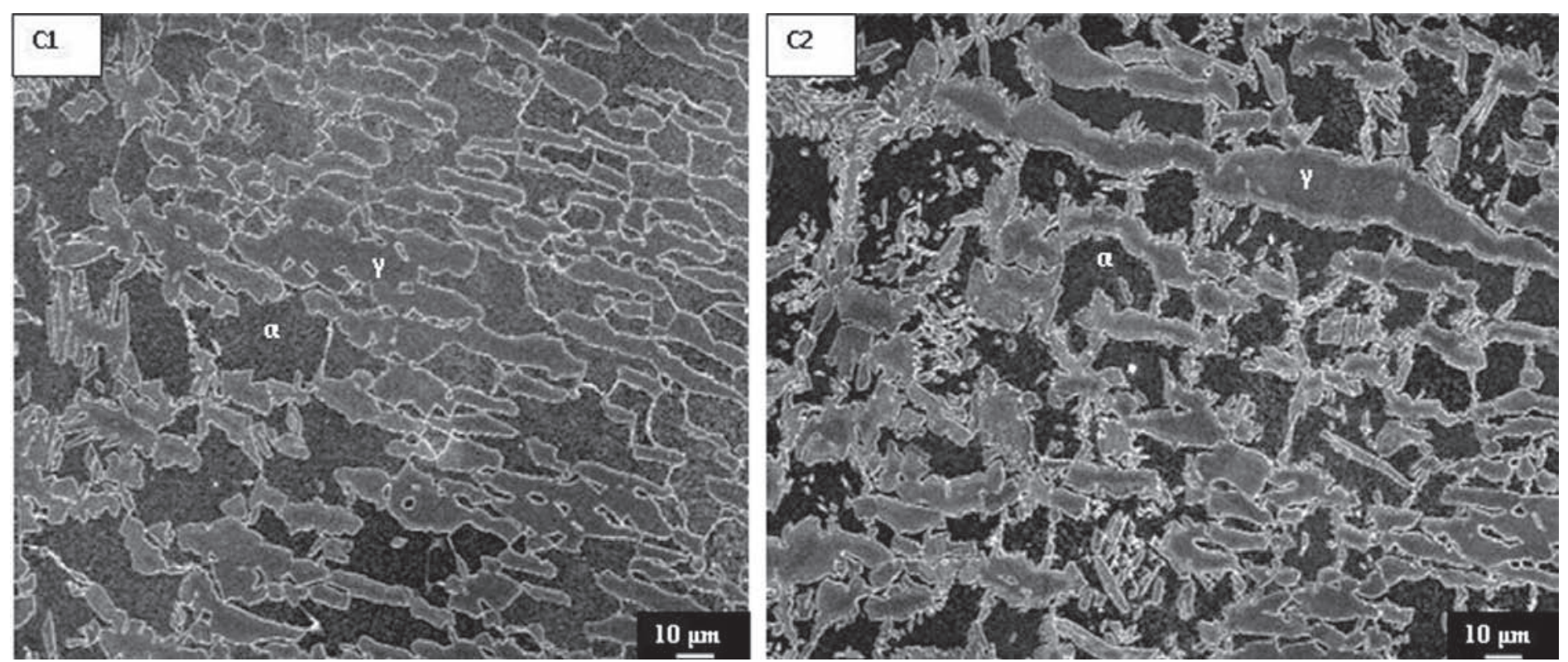

Figura 5. Microestructura de la ZAT en las uniones a) C1 y b) C2. La fase obscura corresponde a la fase ferrita y la fase clara, a la fase austenita

La composición química tanto del material base como del material de aporte fue obtenida en los laboratorios de Metaltest, Inc. y se muestra en la Tabla 4, donde se puede notar que tienen un bajo contenido de $\mathrm{C}$ y altos contenidos de $\mathrm{Cr}$, Mo y $\mathrm{N}$, característico de los aceros inoxidables superdúplex.

Los cambios en la microestructura del acero inoxidable superdúplex SAF 2507 debido a los ciclos térmicos experimentados durante el proceso de soldadura se muestran en la Tabla 5. El material en su condición inicial tiene un balance microestructural de 54\% ferrita-46\% austenita. Sin embargo, el porcentaje de ferrita incrementó en la ZAT de la unión C2 y tuvo una disminución en la unión C1. El mayor porcentaje de ferrita se observó en la unión C2 tanto en la ZAT con un 57\%, como en el cordón de soldadura con un $67 \%$.

El incremento en el porcentaje de ferrita en la ZAT de la unión $\mathrm{C} 2$, está en relación a la temperatura pico alcanzada en el material y a la transformación ferrítica ya que a elevadas temperaturas, aproximadante $1350^{\circ} \mathrm{C}$, la microestructura de la aleación superdúplex es aproximadamente $100 \%$ ferrítica [6]. La temperatura pico para $\mathrm{C} 2$ fue de $1185^{\circ} \mathrm{C}$ mientras que para $\mathrm{C} 1$, fue de $1060^{\circ} \mathrm{C}$. A mayor temperatura pico, mayor es la transformación de austenita-ferrita durante el calentamiento, por lo que el porcentaje de ferrita en la unión C2 es mayor al porcentaje observado en $\mathrm{C} 1$, transformación que toma lugar al ocurrir la disolución de la austenita, la cual está controlada por la difusión de $\mathrm{N}$ en la fase ferrita [10].

La Figura 5 muestra la microestructura de las uniones soldadas $\mathrm{C} 1$ y $\mathrm{C} 2$ de la ZAT donde se observan únicamente las fases ferrita y austenita. El tiempo calculado de enfriamiento en el rango de $1200-800^{\circ} \mathrm{C}$ fue de $1.1 \mathrm{~s}$, para una temperatura pico de $1060^{\circ} \mathrm{C}$ para $\mathrm{C} 1$ mientras que para $\mathrm{C} 2$, el tiempo de enfriamiento calculado fue de $1.4 \mathrm{~s}$ con una temperatura pico de $1185^{\circ} \mathrm{C}$, lo cual indica que el ciclo térmico experimentado en la ZAT del acero inoxidable superdúplex, no es suficiente para promover la precipitación de fase sigma durante la aplicación del proceso de soldadura GTAW, incluso tomando en consideración los recalentamientos en dicha zona a causa de los cordones depositados, ya que se ha establecido que la fase sigma se forma en el rango de $600-900^{\circ} \mathrm{C}$ aproximadamente [11], lo cual es corroborado por Palmer et al. [8], quien reporta que la fase sigma se forma a $850^{\circ} \mathrm{C}$ con un tiempo mínimo de 40 segundos, lo que explicaría su ausencia en este caso.

La Figura 6 muestra la microestructura de la unión soldada $\mathrm{C} 3$ en donde se observa claramente el cambio microestructural 


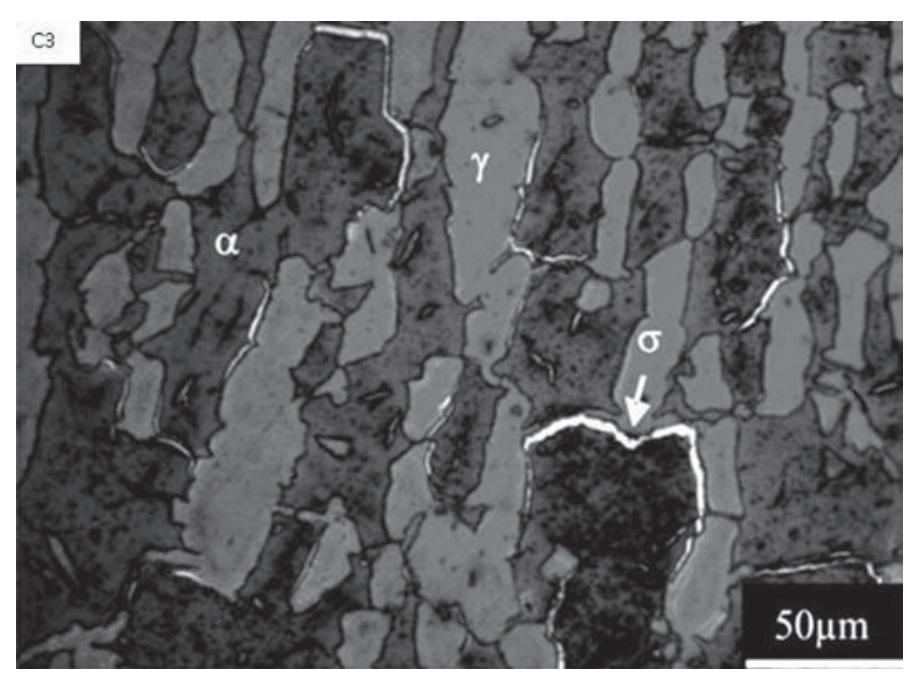

Figura 6. Microestructura de la ZAT en la unión soldada C3. La fase obscura corresponde a la fase ferrita, la fase clara a la fase austenita y la fase brillante, corresponde a la fase sigma.

durante la aplicación del proceso de soldadura al depositarse los cordones consecutivamente con la temperatura entre pasos de $400-450^{\circ} \mathrm{C}$. En la ZAT se observó la presencia de fase sigma y por lo tanto, la disminución de la fase ferrita, mientras que en el cordón de soldadura únicamente se observan austenita y ferrita.

La disminución de fase ferrita en la zona afectada térmicamente se debe a que dicha zona experimenta una permanencia a temperaturas por debajo de la línea sólidus a consecuencia de los pasos de soldadura depositados, por lo que existe suficiente tiempo para que tome lugar la transformación ferrita-austenita justo después de la solidificación. La transformación austenitaferrita que ocurre durante el calentamiento, al estar controlada por la difusión de $\mathrm{N}$ al igual que la transformación ferrita-austenita durante el enfriamiento rápido, se considera reversible [10] por lo que, tomando en cuenta el recalentamiento experimentado en la ZAT por los pasos de soldadura, durante el enfriamiento, se promueve de nuevo la transformación ferrita-austenita, siendo la austenita, fase mayoritaria a temperatura ambiente. Si bien la disminución de ferrita se puede atribuir a la precipitación de fase sigma, no se considera en este caso debido a que el porcentaje presente de fase sigma es pequeño en comparación al porcentaje remanente de ferrita. La precipitación de fase sigma en la ZAT ocurre debido a la permanencia a elevadas temperaturas como son las temperaturas pico experimentadas a causa de los pasos de soldadura depositados, ya que se ha reportado que la fase sigma se forma en el rango de $600-950^{\circ} \mathrm{C}$, siendo su precipitación más rápida entre 700 y $900^{\circ} \mathrm{C}[16]$.

Los resultados indican que la fase ferrita se transforma en austenita y fase sigma mediante la reacción eutectoide ferrita à austenita + sigma [11] a causa de los ciclos térmicos generados por el proceso de soldadura GTAW. Debido a que la fase sigma crece a expensas de la ferrita y debido a que la difusión del $\mathrm{N}$ en la fase ferrita propicia la transformación ferrita-austenita, la disminución de ferrita es inminente.

La fase sigma precipita en las interfases ferrita-ferrita o ferrita-austenita [11]. Los resultados muestran que la fase sigma

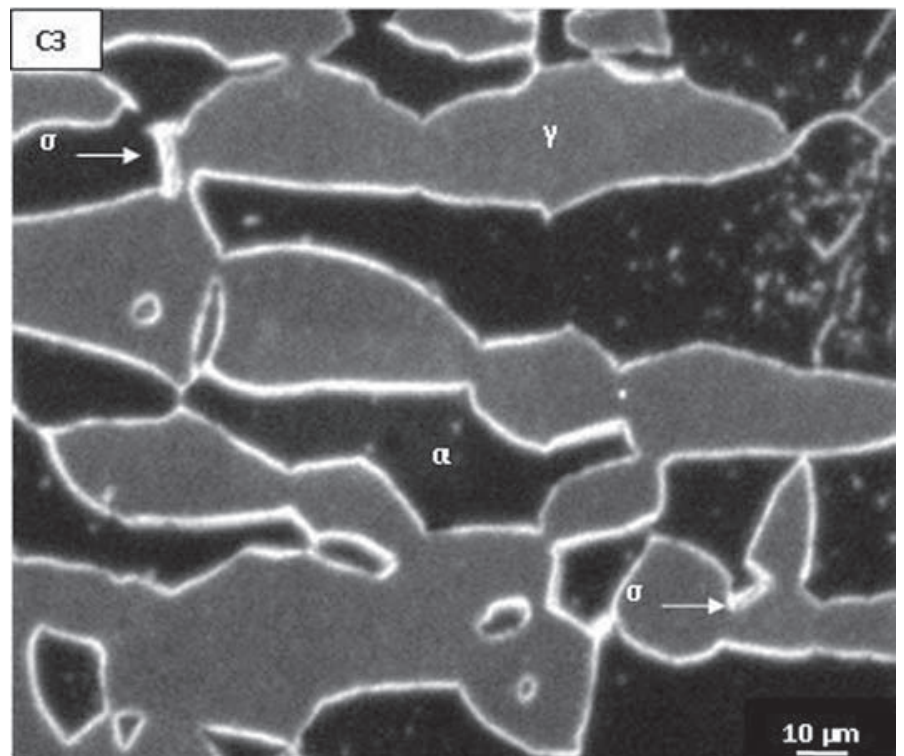

Figura 7. Microestructura de la ZAT en la unión soldada C2. La fase obscura corresponde a la fase ferrita, la fase clara a la fase austenita y la fase brillante rodeando los límites de granos ferrita-austenita, corresponde a la fase sigma.

precipitó en ambas interfases, pero principalmente en la interfase ferrita-ferrita.

La fase sigma está compuesta por elementos alfágenos, principalmente por $\mathrm{Cr}$ y Mo, y por lo tanto, su formación promueve zonas con bajo contenido de dichos elementos, lo que puede ocasionar una disminución de la resistencia a la corrosión localizada [17].

La Figura 7 muestra la fase sigma precipitada en el límite de grano ferrita-austenita en la ZAT de la unión soldada C3. En los microanálisis mostrados en la Tabla 6 de las fases ferrita, austenita y sigma, se observa un mayor contenido de Mo en la fase ferrita de la unión C3 en comparación a las uniones C1 y $\mathrm{C} 2$, indicando una sobresaturación de Mo en la fase ferrita, lo que promovería el inicio de la precipitación de fase sigma, la cual tiene un contenido de Cr, Mo y Si mayor en comparación a las fases ferrita y austenita de las uniones $\mathrm{C} 1$ y $\mathrm{C} 2$.

Bajo el procedimiento C3 el porcentaje de ferrita en el MS es mayor pero menor en la ZAT en comparación a lo observado en los procedimientos $\mathrm{C} 1$ y C2 (Tabla 3), lo cual se atribuye a la temperatura entre pasos $\left(400^{\circ} \mathrm{C}-450^{\circ} \mathrm{C}\right)$, lo que da lugar a una temperatura pico mayor y a un enfriamiento más lento en esa zona. Como se mencionó anteriormente, la microestructura es aproximadamente $100 \%$ ferrita a elevadas temperaturas y debido a que las temperaturas elevadas se mantienen por la deposición consecutiva de los cordones de soldadura, la microestructura al solidificar y enfriar, mantiene un mayor porcentaje de la fase ferrita en la zona del MS, aunado a esto, la deposición consecutiva de los cordones de soldadura, promovió la precipitación de la fase sigma en la ZAT, al permanecer en el rango de temperaturas de formación de fase sigma.

Si bien el porcentaje de fase sigma en la probeta C3 no es significativamente elevado, ésta se encuentra localizada 
Tabla 6. Composición química de las fases ferrita, austenita y sigma en las uniones C1, C2 y C3.

\begin{tabular}{|c|c|c|c|c|c|c|c|c|c|c|c|c|c|}
\hline \multicolumn{4}{|c|}{$\mathrm{Cr}$} & \multicolumn{2}{|c|}{$\mathrm{Ni}$} & \multicolumn{2}{|c|}{ Mo } & \multicolumn{2}{|c|}{$\mathrm{Mn}$} & \multicolumn{2}{|c|}{$\mathrm{Si}$} & \multicolumn{2}{|c|}{$\mathrm{Fe}$} \\
\hline & Fase & ZAT & CS & ZAT & CS & ZAT & CS & ZAT & CS & ZAT & $\mathrm{CS}$ & ZAT & CS \\
\hline & $\gamma$ & 21.7 & 22.4 & 8.1 & 8.66 & 4.4 & 4.4 & 1.4 & 0.47 & 0.3 & 0.29 & 61.5 & 56.9 \\
\hline C) & $\alpha$ & 21.8 & 21.3 & 6.9 & 9.26 & 14.5 & 14.5 & 0.9 & 0.38 & 0.3 & 0.28 & 53.2 & 52.0 \\
\hline & $\sigma$ & 22.1 & -- & 6.8 & -- & 5.2 & -- & 1.2 & -- & 0.6 & -- & 60 & -- \\
\hline
\end{tabular}

\begin{tabular}{|c|c|c|c|c|c|c|c|c|c|c|c|c|c|}
\hline \multicolumn{4}{|c|}{$\mathrm{Cr}$} & \multicolumn{2}{|c|}{$\mathrm{Ni}$} & \multicolumn{2}{|c|}{ Mo } & \multicolumn{2}{|c|}{$\mathrm{Mn}$} & \multicolumn{2}{|c|}{$\mathrm{Si}$} & \multicolumn{2}{|c|}{$\mathrm{Fe}$} \\
\hline & Fase & ZAT & CS & ZAT & $\mathrm{CS}$ & ZAT & $\mathrm{CS}$ & ZAT & CS & ZAT & CS & ZAT & CS \\
\hline & $\gamma$ & 20.3 & 21.5 & 7.4 & 7.5 & 3.0 & 3.5 & 0.6 & 0.39 & 0.3 & 0.32 & 57.7 & 54.6 \\
\hline ? & $\alpha$ & 22.0 & 21.1 & 5.5 & 7.9 & 6.3 & 7.4 & 0.49 & 0.34 & 0.3 & 0.34 & 55.1 & 52.5 \\
\hline $\mathrm{C3}$ & $\sigma$ & -- & -- & -- & -- & -- & -- & -- & -- & -- & --- & -- & -- \\
\hline
\end{tabular}

\begin{tabular}{|c|c|c|c|c|c|c|c|c|c|c|c|c|c|}
\hline \multicolumn{4}{|c|}{$\mathrm{Cr}$} & \multicolumn{2}{|c|}{$\mathrm{Ni}$} & \multicolumn{2}{|c|}{ Mo } & \multicolumn{2}{|c|}{$\mathrm{Mn}$} & \multicolumn{2}{|c|}{$\mathrm{Si}$} & \multicolumn{2}{|c|}{$\mathrm{Fe}$} \\
\hline \multirow{4}{*}{ C1 } & Fase & ZAT & CS & ZAT & CS & ZAT & CS & ZAT & CS & ZAT & CS & ZAT & CS \\
\hline & $\gamma$ & 21.8 & 22.04 & 6.2 & 8.4 & 3.6 & 3.7 & 0.7 & 0.34 & 0.2 & 0.29 & 58.7 & 56.8 \\
\hline & $\alpha$ & 21.8 & 23.6 & 6.5 & 7.0 & 9.7 & 8.8 & 0.6 & 0.4 & 0.2 & 0.3 & 56.6 & 54.6 \\
\hline & $\sigma$ & -- & -- & -- & -- & -- & -- & -- & -- & -- & -- & -- & -- \\
\hline
\end{tabular}

en los límites de grano ferrita/austenita de la ZAT. Durante la transformación de ferrita a austenita, la ferrita se enriquece de Cr y Mo (ver Tabla 4), volviéndose el sitio preferencial para la formación de fase sigma. La transformación ferrita-sigma compromete significativamente la resistencia a la corrosión de la aleación debido a que las zonas adyacentes a la fase sigma, quedan empobrecidas de $\mathrm{Cr}$ y Mo, siendo las zonas más susceptibles a la corrosión.

Las mediciones de microdureza mostraron que para las tres uniones soldadas $\mathrm{C} 1, \mathrm{C} 2$ y $\mathrm{C} 3$, la fase austenita tiene una dureza mayor en la zona del cordón de soldadura que en la ZAT, atribuyéndose a la alta solubilidad de $\mathrm{N}$ en la fase austenita, lo cual permite el acomodamiento de los átomos de N. La tabla 7 muestra las mediciones de microdureza en los granos de fase ferrita y fase austenita. En cuanto a la fase ferrita, la dureza se ve disminuida en la zona del cordón de soldadura en comparación a la ZAT en las uniones C1 y C3, mientras que para la unión $\mathrm{C} 2$, la ferrita tuvo un incremento en la dureza de la zona del cordón de soldadura en comparación a la ZAT, posiblemente por la sobresaturación de elementos intersticiales como el $\mathrm{C}$ y $\mathrm{N}$ en la fase ferrita.

Tabla 7. Microdureza de las fases ferrita y austenita en las uniones soldadas

\begin{tabular}{|c|c|c|c|}
\hline \multicolumn{2}{|c|}{ FASE } & ZAT (Vickers) & CS (Vickers) \\
\hline \multirow{3}{*}{ C1 } & $\gamma$ & 360 & 398 \\
\cline { 2 - 4 } & $\alpha$ & 354 & 351 \\
\hline \multirow{3}{*}{ C3 } & $\gamma$ & 354 & 387 \\
\cline { 2 - 4 } & $\alpha$ & 268 & 255 \\
\hline \multirow{2}{*}{ C2 } & $\gamma$ & 351 & 390 \\
\cline { 2 - 4 } & $\alpha$ & 213 & 297 \\
\hline
\end{tabular}

\section{Conclusiones}

1. No se observó la precipitación de fase sigma bajo las temperaturas de interpasos de $25-30^{\circ} \mathrm{C}$ y $100-150^{\circ} \mathrm{C}$ con un tiempo de permanencia de 1.1 y 1.4 segundos respectivamente debido a las bajas temperaturas y los cortos tiempos de permanencia a dichas temperaturas.

2. La precipitación de fase sigma fue evidente bajo la temperatura de interpasos de $400-450^{\circ} \mathrm{C}$, sin embargo, el bajo porcentaje de fase sigma se debe al corto tiempo de permanencia en comparación al porcentaje reportado por Palmer et.al, en donde los tiempos de permanencia fueron prolongados $\mathrm{y}$ controlados mediante la aplicación de tratamientos térmicos.

3. La fase sigma es observada en los límites de grano ferritaferrita debido a que está formada por $\mathrm{Cr}$ y Mo los cuales son elementos alfágenos y lo que provoca la disminución de ferrita al formarse la fase sigma.

\section{Agradecimientos}

Los autores agradecen a CONACYT por su apoyo financiero así como a SANDVIK de México por el material empleado para la presente investigación.

\section{Referencias Bibliográficas}

1. BALANCIN, O., HOFFMANN, W.A.M., JONAS, J.J. Influence of microstructure on the flow behavior of dúplex stainless steels at high temperatures, Metallurgical and Materials Transactions, v.31A, n5, p.1353-1363, May. 2000.

2. ASM Specialty Handbook. Stainless Steel, Materials Park, OH, 1999, p.32-34.

3. STEVENS, K.J. Fatigue performance and microanalysis of heat treated 2205 duplex stainless steel, Materials Science and Technology, v.15, n.8, p.903-908, Ago. 1999.

4. KURODA T. Role of sigma phase on hydrogen embrittlement of super duplex stainless steels, Joining and Welding Research Institute, v.34, n.2, p.63-68, 2005.

5. DENG, B., WANG, Z., JIANG, Y., SUN, T., XU, J., LI, J. Effect of thermal cycles on the corrosion and mechanical properties of UNS S31803 duplex stainless steel, Corrosion Science, v.51, p.2969-2975, 2009.

6. RAMIREZ, A.J., LIPPOLD, J.C., BRANDI, S.D. The relationship between chromium nitride and secondary austenite precipitation in duplex stainless steel, Metallurgical and Materials Transactions, v. 34A, n.8, p. 1575-1597, Ago.2003.

7. STRADOMSKI, Z., DYJA, D. Sigma phase precipitation in duplex phase stainless steels, ProQuest, 2009. 
8. PALMER, T.A, ELMER, J.W, BABU, S.S., SPECHT, E.D. Direct observations of sigma phase growth and dissolution in 2205 duplex stainless steel, Trend in Welding Research, Pine Mountain, GA, United States, 16-20 de Mayo 2005.

9. OTAROLA, T., HOLLNER, S., BONNEFOIS, B., ANGLADA, M., COUDREUSE, L., MATEO, A. Embrittlement of a superduplex stainless steel in the range of $550-700^{\circ} \mathrm{C}$, Engineering Failure Analysis, v.12, p.930$941,2005$.

10. HEMMER, H., GRONG, O. A process model for the heataffected zone microstructure evolution in duplex stainless steel weldments: Part I. the model, Metallurgical and Materials Transactions, v.30A, n.11, p.2915-2929, Nov. 1999.

11. LIPPOLD, J.C, KOTECKI, D. Welding Metallurgy and Weldability of Stainless Steels, 2005.

12. Welding guide for Sandvik duplex stainless steel, Suecia, 2003.

13. EASTERLING, K. Introduction to the physical metallurgy of welding, $2^{\text {nd }}$ edition, Butterworths, 1992.

14. SIEURIN, H., SANDSTRÖM, R. Austenite reformation in the heat-affected zone of dúplex stainless steel 2205, Materials Science and Engineering A, v.418, p.250-256, 2006.

15. GRONG, O. Metallurgical modeling of welding, London: The Institute of Materials, $1^{\text {st }}$ edition, 1994.

16. KOBAYASHI, D.Y., WOLYNEC, S. Evaluation of the low corrosion resistant phase formed during the sigma phase precipitation in duplex stainless steels, Materials Research, v.2, n.4, p.239-247, 1999.

17. MAGNABOSCO, R., ALONSO-FALLEIROS, N. Pit morphology and its relation to microstructure of $850^{\circ} \mathrm{C}$ aged duplex stainless steel, Corrosion, v.61, n.2, p. 130-136, Feb. 2005. 